\title{
Accounting for the Economic Risk Caused by Variation in Disease Severity in Fungicide Dose Decisions, Exemplified for Mycosphaerella graminicola on Winter Wheat
}

\author{
D. E. te Beest, N. D. Paveley, M. W. Shaw, and F. van den Bosch
}

First and fourth authors: Biomathematics and Bioinformatics, Rothamsted Research, Harpenden, Hertfordshire, AL5 2 JQ, UK; first author: Centre for Infectious Diseases Control, National Institute for Public Health and the Environment (RIVM), Bilthoven, the Netherlands; second author: ADAS High Mowthorpe, Duggleby, Malton, North Yorkshire, YO17 8BP, UK; and third author: School of Biological Sciences, University of Reading, Reading RG6 6AS, UK. Accepted for publication 25 January 2013.

\begin{abstract}
te Beest, D. E., Paveley, N. D., Shaw, M. W., and van den Bosch, F. 2013. Accounting for the economic risk caused by variation in disease severity in fungicide dose decisions, exemplified for Mycosphaerella graminicola on winter wheat. Phytopathology 103:666-672.

A method is presented to calculate economic optimum fungicide doses accounting for the risk aversion of growers responding to variability in disease severity between crops. Simple dose-response and disease-yield loss functions are used to estimate net disease-related costs (fungicide cost plus disease-induced yield loss) as a function of dose and untreated severity. With fairly general assumptions about the shapes of the probability distribution of disease severity and the other functions involved, we show that a choice of fungicide dose which minimizes net costs, on average, across seasons results in occasional large net costs caused by

surance. For example, a grower may decide to accept "high-loss" years 1 year in 10 or 1 year in 20 (i.e., specifying a proportion of years in which disease severity and net costs will be above a specified level). Our analysis shows that taking into account disease severity variation and risk aversion will usually increase the dose applied by an economically rational grower. The analysis is illustrated with data on Septoria tritici leaf blotch of wheat caused by Mycosphaerella graminicola. Observations from untreated field plots at sites across England over 3 years were used to estimate the probability distribution of disease severities at mid-grain filling. In the absence of a fully reliable disease forecasting scheme, reducing the frequency of high-loss years requires substantially higher doses to be applied to all crops. Disease-resistant cultivars reduce both the optimal dose at all levels of risk and the disease-related costs at all doses.
\end{abstract} inadequate control in high disease seasons. This may be unacceptable to a grower with limited capital. A risk-averse grower can choose to reduce the size and frequency of such losses by applying a higher dose as in-
Additional keywords: disease risk.
Foliar-applied fungicides are used to control diseases of a wide range of crops. The total fungicide dose applied during a cropping season may be varied by altering the spray interval or by adjusting the dose applied per spray application. The economic optimum fungicide dose for a particular field and year is that dose which minimizes the combined cost of fungicide sprays and disease-induced loss of marketable product. It has been argued that growers use higher doses than are justified by the resulting increases in yield $(5,17)$. However, the economically optimum dose depends on disease severity, which varies greatly between seasons. If disease is severe and a fungicide dose is used that is optimal under average conditions, a grower will suffer a large financial loss in that year. They may be willing to pay the additional cost of a higher fungicide dose each year as a risk-reducing investment (i.e., "insurance") in order to be protected from occasional large costs. Hence, growers may not necessarily aim to maximize their mean gross margin over the years. Instead, they may aim to minimize the risk of a severe financial loss which might threaten their business. By quantifying the relationship between risk and dose, advice on optimal fungicide dose could take into account this risk aversion.

Corresponding author: F. van den Bosch;

E-mail address: frank.vandenbosch@ rothamsted.ac.uk

http://dx.doi.org/10.1094/PHYTO-05-12-0119-R

(C) 2013 The American Phytopathological Society
This article develops a method by which optimal fungicide dose can be calculated for a predefined level of risk, if the distribution of disease severity between years is known. This allows the dose to be identified at which risk is reduced to a level acceptable to a particular grower. The method developed is exemplified with the case of Mycosphaerella graminicola (Septoria tritici leaf blotch $[\mathrm{STB}]$ ) on winter wheat, which is one of the main drivers of fungicide use on this crop in the United Kingdom $(3,5)$.

\section{THEORY AND APPROACHES}

Disease data. The disease data used to quantify the probability distribution of severity over years were from the Crop Monitor program (www.cropmonitor.co.uk), in which disease severity was assessed each year in replicated field plots of winter wheat at a range of sites across England. This data set has been analyzed previously, as described by te Beest et al. (15), but sites that had to be excluded in that previous article (because there was no nearby weather station available) were included in the present analysis. The data cover the years 2003 to 2005 and 18 experimental sites; in total, 44 year-site combinations (Table 1). In each year-site, observations were made on cultivars that differed in their resistance to $M$. graminicola. The observations were categorized into a resistant and a susceptible group according to the resistance ratings of the cultivars (1). Ratings were on a scale of 1 to 9 , with 1 being very susceptible and 9 very resistant. The highest resistance rating in the data set was 7 and the lowest was 3. A resistant group was defined that consisted of cultivars with ratings 
6 and 7 and a susceptible group of cultivars with ratings 3 , 4, and 5. Disease severity on leaf 2 at growth stage (GS) 75 (medium milk stage of grain maturity), which has been used as an indicator for likely yield loss $(6,16)$, was used here to summarize the observations (Table 2). All observations were from plots untreated with fungicides.

In commercial practice, a grower would want to control risk for their particular site and the cultivars they grow. However, for the analysis presented here, there is a trade-off between the specificity of the data set to a particular location and cultivar and the size of the data set (and, hence, the reliability of parameters estimated from it). Hence, the data were subdivided into susceptible and resistant cultivars but not into high- or low-risk regions of England. Nevertheless, the results should provide an acceptable approximation for most locations, given that seasonal STB variation is greater than regional variation (4).

The active substance epoxiconazole (as the commercial product Opus; BASF) was used as an example fungicide with efficacy against $M$. graminicola. One full fungicide dose (Opus at 1 liter/ha) is one recommended manufacturer's dose as described by Finney (2). The product label limits the maximum total dose applied in any one season to 2 liters/ha. In the United Kingdom, typical practice is to apply fungicide at approximately GS 31 or 32 (first or second node detectable, typically mid-April), at GS 39 (flag leave visible, typically end of May), and at GS 59 (emergence head complete, typically mid-June).

Disease risk. The probability that disease severity $S$ at GS 75 will develop in a given year-site was summarized by fitting an exponential distribution by maximum likelihood to the disease severity data. Therefore, the probability density of $S, p(S)$, obeyed

$$
p(S)=\alpha e^{-\alpha S}
$$

where $1 / \alpha$ is the mean disease severity. Larger values of $\alpha$ thus correspond to smaller probabilities of severe disease. Fitting was

TABLE 1. Overview of the years and sites used to derive the distribution of severities $^{\mathrm{a}}$

\begin{tabular}{lccc}
\hline Site & 2003 & 2004 & 2005 \\
\hline Andover & $\ldots$ & $\mathrm{X}$ & $\mathrm{X}$ \\
Ashford & $\mathrm{X}$ & $\mathrm{X}$ & $\mathrm{X}$ \\
Askham Bryan & $\mathrm{X}$ & $\mathrm{X}$ & $\ldots$ \\
Boxworth & $\mathrm{X}$ & $\mathrm{X}$ & $\mathrm{X}$ \\
Bridgets & $\mathrm{X}$ & $\ldots$ & $\ldots$ \\
Cirencester & $\ldots$ & $\mathrm{X}$ & $\mathrm{X}$ \\
Drayton & $\mathrm{X}$ & $\mathrm{X}$ & $\mathrm{X}$ \\
Exeter & $\mathrm{X}$ & $\mathrm{X}$ & $\mathrm{X}$ \\
Gleadthorpe & $\mathrm{X}$ & $\mathrm{X}$ & $\mathrm{X}$ \\
High Mowthorpe & $\mathrm{X}$ & $\mathrm{X}$ & $\mathrm{X}$ \\
Kirton & $\ldots$ & $\mathrm{X}$ & $\mathrm{X}$ \\
Lavenham & $\mathrm{X}$ & $\mathrm{X}$ & $\mathrm{X}$ \\
Morley & $\mathrm{X}$ & $\mathrm{X}$ & $\mathrm{X}$ \\
Newcastle & $\ldots$ & $\ldots$ & $\mathrm{X}$ \\
Rosemaund & $\mathrm{X}$ & $\mathrm{X}$ & $\ldots$ \\
Tadcaster & $\mathrm{X}$ & $\ldots$ & $\mathrm{X}$ \\
Terrington & $\mathrm{X}$ & $\ldots$ & $\ldots$ \\
Wye & $\mathrm{X}$ & $\ldots$ & $\mathrm{X}$ \\
York & $\ldots$ & $\mathrm{X}$ &
\end{tabular}

${ }^{\mathrm{a}}$ Data were available from site-year combinations indicated with an $\mathrm{X}$. done with Genstat (11) over all cultivars (Fig. 1A) and separately over resistant cultivars (Fig. 1B) and susceptible cultivars (Fig. 1C). A Kolmogorov-Smirnov goodness of fit test was used (11) to test whether the data differed significantly from the fitted exponential distribution.

Disease costs and economic best optimum dose. The cost attributable to disease, $C_{M}$, consists of (i) the costs of the application of fungicides, $C_{F}$, and (ii) the costs incurred due to diseaseinduced yield loss, $C_{Y}$. Both costs depend on the fungicide dose used, $D$; therefore, we can write

$$
C_{M}(D)=C_{F}(D)+C_{Y}(D)
$$

The cost of fungicide treatment is the product of fungicide dose and the fungicide price per unit dose, $P_{D}(\mathfrak{f})$, resulting in $C_{F}(D)=$ $D \times P_{D}$. The cost of yield loss, $C_{Y}(D)$, is calculated as the product of the price of the crop $\left(P_{W}\right)$ ( $£$ per metric ton), the yield in the absence of disease ( $Y$, metric tons per hectare), and the fraction of the yield lost due to the disease $\left(Y_{L}\right)$.

The yield loss $\left(Y_{L}\right)$ is a function $L \times g[S \times f(D)]$, where $S$ is the disease severity (measured appropriately for the pathosystem) in a season where no fungicide was applied, $[1-f(D)]$ is the control achieved when fungicides are applied at dose $D, L$ is the proportion of yield lost when severity is at its maximum value of $100 \%$, and $g$ is the function that relates intermediate values of severity to a proportion of yield lost. In the simplest case, yield loss is linearly related to disease severity:

$$
Y_{L}(D, S)=L \times S \times f(D)
$$

but other relations are easily substituted in equation 3 . In the sensitivity analysis (Table 3), we test the effect of alternative relationships.

Within the range of doses around an economic optimum, any plausible function to model the fungicide dose response curve would have to be monotonically decreasing towards some asymptote and have a steadily decreasing slope. Therefore, following Paveley et al. (8-10), the fraction of the severity remaining after fungicide application at dose $D$ is modeled by

$$
f(D)=1-R_{D}+R_{D} \times \exp (-k \times D)
$$

The parameter $R_{D}$ is the proportional control if a very large dose is used and the parameter $k$ defines the curvature of the dose response curve. Combining these relationships, we obtain

$$
\begin{aligned}
C_{M}(D, S)= & D \times P_{D}+L \times S \times\left[1-R_{D}+R_{D} \times\right. \\
& \exp (-k \times D)] \times P_{W} \times Y
\end{aligned}
$$

Clearly, the disease severity that would develop if no fungicides were applied, $S$, differs between years (Fig. 1). The mean cost,

\begin{tabular}{|c|c|c|c|c|c|}
\hline Cultivars & Mean severity (\%) & Number of observations & Year-sites & $\alpha^{\mathrm{a}}$ & $P^{\mathrm{a}}$ \\
\hline All & 11 & 146 & 44 & 8.8 & 0.14 \\
\hline Resistant & 7 & 76 & 31 & 14.2 & 0.11 \\
\hline Susceptible & 16 & 70 & 42 & 6.2 & 0.70 \\
\hline
\end{tabular}
averaging the costs over a long run of years where dose $D$ is applied every year, can be calculated from

$$
\begin{aligned}
\mathrm{E}\left[C_{M}(D)\right]= & D \times P_{D}+L \times \mathrm{E}[S] \times\left[1-R_{D}+R_{D} \times\right. \\
& \exp (-k \times D)] \times P_{W} \times Y
\end{aligned}
$$

TABLE 2. Summary of disease data and exponential probability distributions fitted across all sites and years

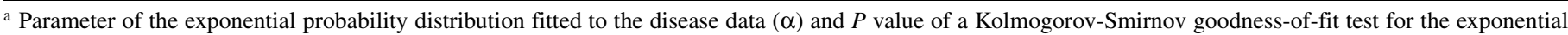
probability distribution fitted to the disease data. None of the fitted distributions is significantly different from the exponential distribution. 

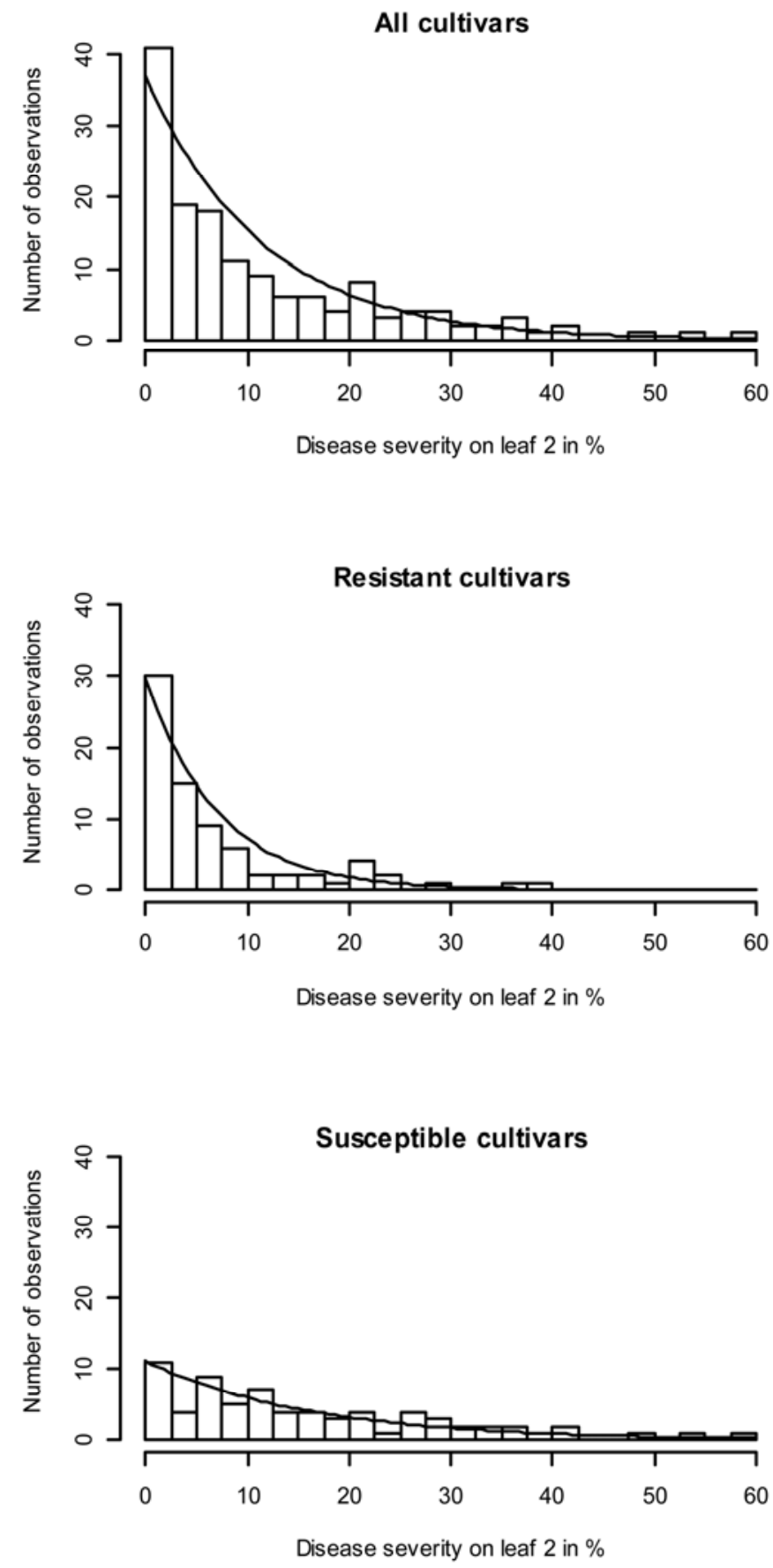

Fig. 1. Histograms of the frequency of Mycosphaerella graminicola severity in crops grown at 44 site-years during 2003 to 2005. Solid line: best-fitting exponential distribution fitted $\left(\alpha \cdot e^{-\alpha S}\right)$. The $\alpha$ and the goodness of fit statistics are given in Table 2. where $\mathrm{E}[S]$ is the severity that develops without fungicides, averaged over years. If the fungicide dose is increased, the costs of fungicide will increase and the costs of disease-induced yield loss will decrease; therefore, there is a minimum to the cost curve. The dose at which the costs are minimal, $D_{E}$, can be calculated by differentiating equation 6 and equating the derivative to zero, to obtain the equation

$$
D_{E}=\frac{-\log \left(\frac{P_{D}}{L \times \mathrm{E}[S] \times k \times R_{D} \times P_{W} \times Y}\right)}{k}
$$

Optimum dose accounting for risk. Equations 6 and 7 use the mean severity of an epidemic to derive the dose that minimizes average costs for disease control. Risk-averse growers may aim, instead, to minimize the costs of extreme seasons. The dose required to achieve this was calculated as follows.

We suppose that a grower is only prepared to accept a loss greater than some critical amount-perhaps that amount which could cause some risk to the survival of the business-in a small proportion $K$ of years. Once the critical loss $C_{M K}$ is specified, equation 5 can be rearranged to calculate the equivalent critical severity, $S_{K}$, as a function of the dose, $D$, of fungicide applied:

$$
S_{K}=\frac{C_{M K}-D \times P_{D}}{L \times\left(1-R_{D}+R_{D} \times e^{-k \times D}\right) \times P_{W} \times Y}
$$

If the cumulative probability distribution of severity over seasons is a known function $P(S)$, then $K$ can be calculated as $K=P\left(S_{K}\right)$. It is simplest to explain the procedure assuming a particular parametric form for $P(S)$, such as the exponential distribution for $M$. graminicola introduced above. Other forms could be substituted and we discuss the consequences of various forms in the supplementary information. Assuming that-as in the M. graminicola case-disease severity is exponentially distributed (equation $1)$, then $K$ is

$$
K=\int_{S_{K}}^{\infty} \alpha \times e^{-\alpha \times S} d S=e^{-\alpha \times S_{K}}
$$

where $\alpha$ is the parameter of the exponential probability distribution describing variation in disease from season to season. Substituting equation $8\left(S_{K}\right)$ in equation 9 results in

$$
K=\exp \left(-\alpha \frac{C_{M K}-D \times P_{D}}{L \times\left(1-R_{D}+R_{D} \times e^{-k \times D}\right) \times P_{W} \times Y}\right)
$$

Rearranging this to express $C_{M K}$ as a function of $K$, we obtain

\begin{tabular}{|c|c|c|c|c|c|c|c|}
\hline Parameter & Change & $D_{E}$ & $E\left[C_{M}\left(D_{E}\right)\right]$ & $D_{K}$ & $C_{M K}\left(D_{K}\right)$ & $E\left[C_{M}\left(D_{K}\right)\right]$ & $D_{K}-D_{E}$ \\
\hline Default values & & 0.80 & 29.3 & 1.35 & 44.1 & 35.9 & 0.54 \\
\hline$P_{W}$ or $P$ or $L$ & Increased by $10 \%$ & 0.87 & 31.0 & 1.41 & 46.1 & 37.6 & - \\
\hline$P_{W}$ or $P$ or $L$ & Decreased by $10 \%$ & 0.72 & 27.2 & 1.27 & 41.8 & 33.9 & - \\
\hline$P_{D}$ & Decreased by $10 \%$ & 0.88 & 25.1 & 1.43 & 37.2 & 30.4 & - \\
\hline$R_{D}$ & Decreased to 0.75 & 0.70 & 47.6 & 1.25 & 137.0 & 54.1 & - \\
\hline$k$ & Decreased to 1.4 & 1.10 & 45.9 & 2.20 & 74.3 & 59.1 & 1.09 \\
\hline$R_{D}$ and $k$ & Decreased to 0.75 and 1.4 & 0.90 & 61.6 & 2.00 & 164.7 & 74.8 & 1.09 \\
\hline
\end{tabular}

$$
\begin{gathered}
C_{M K}(D, K)=D \times P_{D}-L \times \\
\left(1-R_{D}+R_{D} \times e^{-k \times D}\right) \times P_{W} \times Y \times[\log (K) / \alpha]
\end{gathered}
$$

TABLE 3. Sensitivity analysis ${ }^{\mathrm{a}}$

a Symbols: - indicates that value did not change; other abbreviations relate to equations $6,7,11$, and 12 . The risk level $K$ was set at 0.01 . Default values for the parameters can be found in Table 4.

b $Y_{L}(D, S)=L \times S^{0.5} \times f(D)$. 
Equation 11 is similar to the equation for expected costs (equation 6) but with the expected severity $\mathrm{E}[S]$ replaced by $-\log (K) / \alpha$. A similar derivation would follow for any parametrically defined probability distribution of disease severity, simply substituting the relevant distribution in equation 8 for the exponential distribution $\mathrm{E}[S]=1 / \alpha$; therefore, equations 11 and 6 are the same when $-\log (K)=\alpha$; that is, when a grower is prepared to tolerate losses above the specified level in $K=e^{-1}=37 \%$ of years.

By differentiating equation 11 with respect to dose $D$, setting the derivative to 0 , and solving the resulting equation for $D$, the

TABLE 4. Parameters used in Figures 2 to 4 and Table $5^{\text {a }}$

\begin{tabular}{|c|c|c|c|}
\hline Variable name & Symbol & Value & Dimension \\
\hline Wheat production & $Y$ & 9.15 & metric ton/ha \\
\hline Wheat price & $P_{W}$ & 100 & $£ /$ metric ton \\
\hline Fungicide price per dose & $P_{D}$ & 25 & £/dose \\
\hline Yield loss coefficient & $L$ & 0.81 & - \\
\hline Dose response factor & $k$ & 2.8 & ha/dose \\
\hline Maximum disease reduction & $R_{D}$ & 0.996 & - \\
\hline
\end{tabular}

a Taken from te Beest et al.(14).
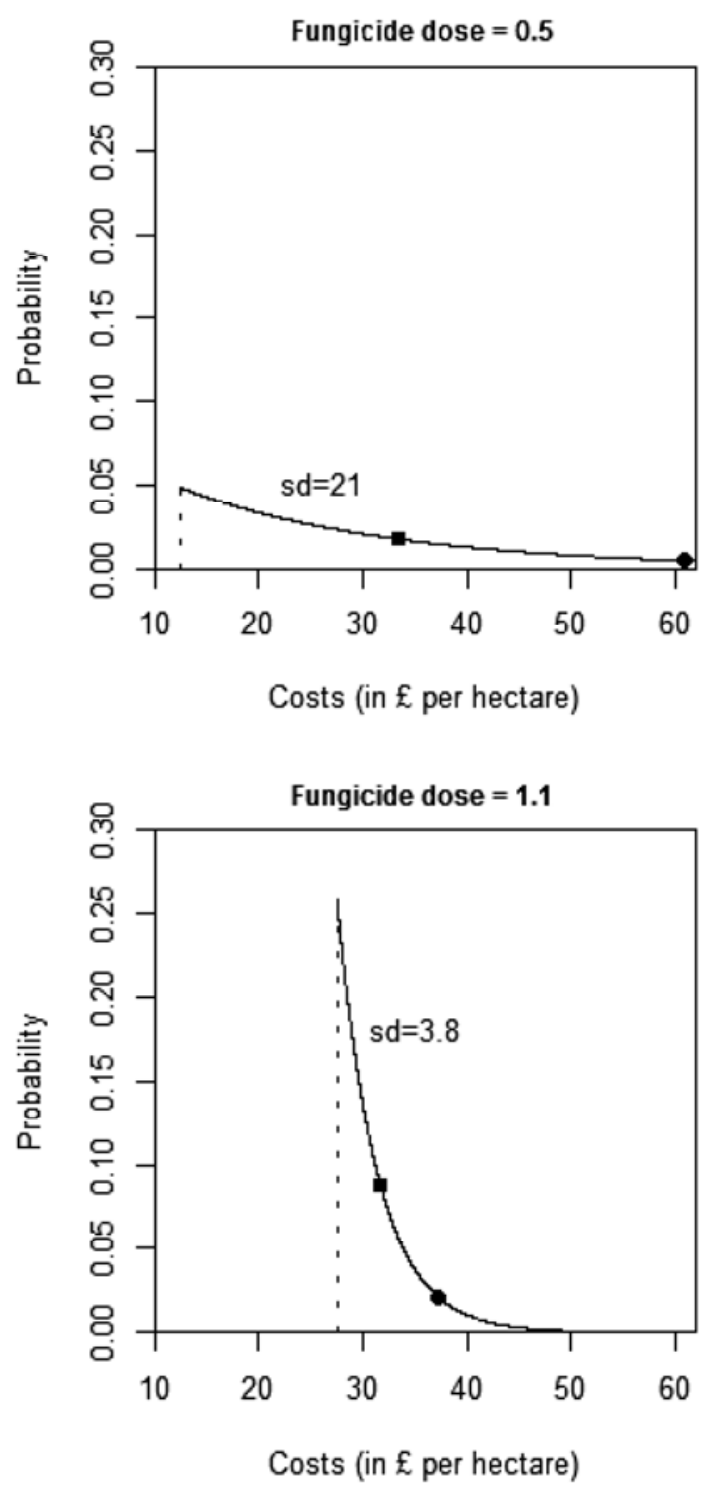

fungicide dose at which $C_{M K}$ is minimal for a given $K, D_{K}$, can be calculated as

$$
D_{K}=\frac{-\ln \left(\frac{-P_{D} \times \alpha}{L \times k \times R_{D} \times P_{W} \times Y \times \log (K)}\right)}{k}
$$

If a grower wishes to reach a particular low risk level, $K_{L}$, from a certain high risk level, $K_{H}$, then, for a severity distribution approximated by an exponential, the difference between the dose providing a risk of $K_{L}\left(D_{K L}\right)$ and the dose providing a risk of $K_{H}$ $\left(D_{K H}\right)$ is independent of yield in the absence of disease or the price of the crop. To see this, the difference can be expressed as

$D_{K L}-D_{K H}=\frac{-\log \left(\frac{-P_{D} \times \alpha}{L \times k \times R_{D} \times P_{W} \times Y \times \log \left(K_{L}\right)}\right)}{k}-\frac{-\log \left(\frac{-P_{D} \times \alpha}{L \times k \times R_{D} \times P_{W} \times Y \times \log \left(K_{H}\right)}\right)}{k}$

which simplifies to

$$
D_{K L}-D_{K H}=\frac{1}{k} \log \left(\frac{\log \left(K_{L}\right)}{\log \left(K_{H}\right)}\right)
$$
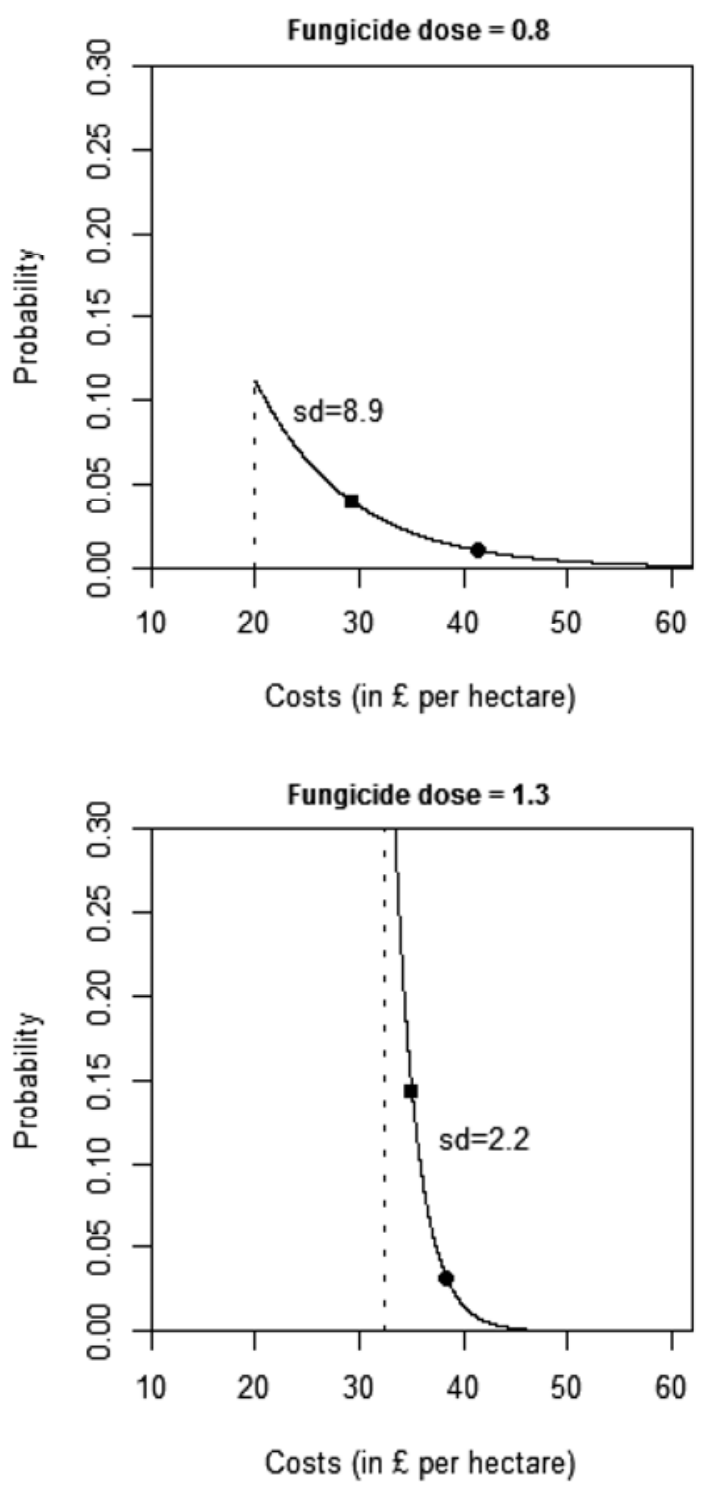

Fig. 2. Probabilities of the net disease-related costs at four fungicide doses, averaged over resistant and susceptible cultivar groupings. The area under each curve represents a probability of one. Standard deviation (sd) in costs associated with each curve is marked on the curve. On each curve, the total probability of costs in excess of the points marked $\bullet$ and are 10 and 37\%, respectively. A farmer prepared to accept higher yield loss in 1 year in 10 should choose the dose which places the $\bullet$ mark as far to the left as possible (in the example, that is the dose of 1.1). 
which depends only on risk levels $K_{L}$ and $K_{H}$ and the dose response parameter $(k)$. However, this property depends on the form of the exponential distribution and is likely not be true in general.

The variance in costs due to the variance in disease severity can be calculated from equation 1 . Assuming that all parameters except the disease severity are constant, we can use the rule that relates the variance in an arbitrary variable $y$ to that in the same variable multiplied by some constant $a, \operatorname{var}(a \cdot y)=a^{2} \cdot \operatorname{var}(y)$ or, rewritten to the standard deviation $s d, s d(a \cdot y)=a \cdot s d(y)$ gives

$$
s d\left(C_{M}\right)=L \times s d(S) \times\left(1-R_{D}+R_{D} \times e^{-k \times D}\right) \times P_{W} \times Y
$$

The parameter values used to illustrate application of the model were estimated in (equation 15) and summarized in (Table 4).

Sensitivity analysis. To test the generality of our conclusions, we examined how robust our results were to changes in the parameter values (Table 3). More broadly, we tested how sensitive our results are to the functional forms that we chose to model

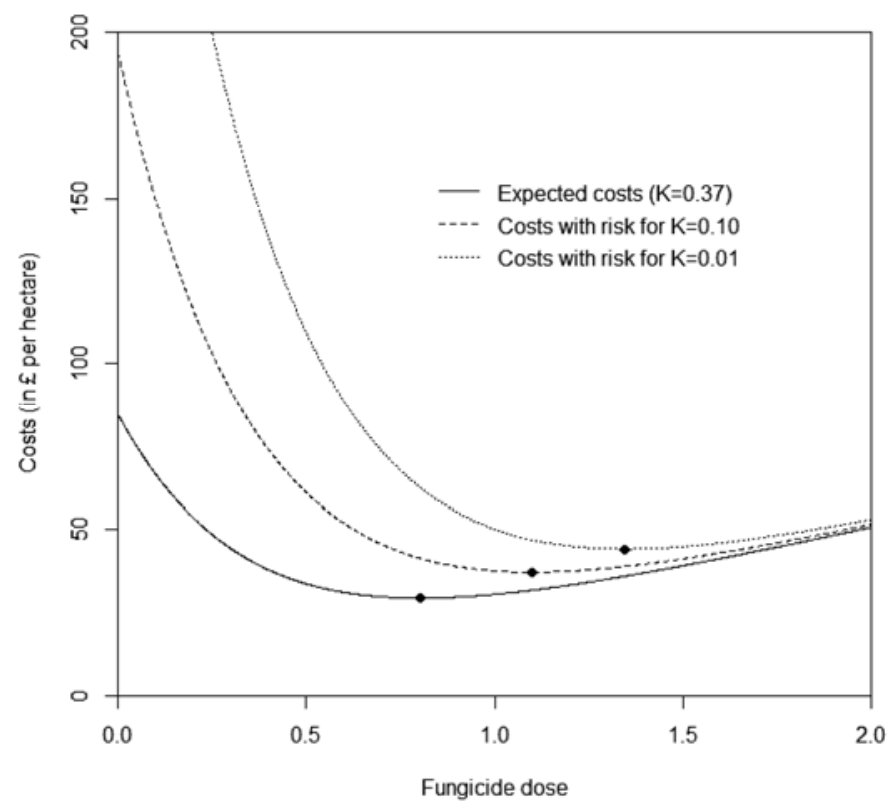

Fig. 3. Minimum long-term average costs $(K=0.37)$ and the costs which will not be exceeded with probability $K$ for $K=0.10$ and $K=0.01$ plotted as a function of the total fungicide dose applied during the season, in liters of the commercial product Opus (active substance, epoxiconazole; BASF) per hectare. Disease severity distribution averaged over susceptible and resistant cultivar groupings. The marks the optimum dose. Cost analyses were done using units of British pounds. These values were converted to American dollars at an exchange rate of 1 pound $=1.60$ dollars.

TABLE 5. Optimal fungicide dose and related costs in relation to acceptable risk, $K$

\begin{tabular}{lcccc}
\hline Cultivars & $K^{\mathrm{a}}$ & Dose & $\mathrm{E}\left(C_{M}\right)^{\mathrm{b}}$ & $C_{M K} \mathrm{c}^{\mathrm{c}}$ \\
\hline All & 0.37 & 0.80 & 29.3 & 29.3 \\
& 0.10 & 1.10 & 31.7 & 37.1 \\
Resistant & 0.01 & 1.35 & 35.9 & 44.1 \\
& 0.37 & 0.63 & 24.9 & 24.9 \\
Susceptible & 0.10 & 0.93 & 27.3 & 32.6 \\
& 0.01 & 1.17 & 31.5 & 39.3 \\
& 0.37 & 0.93 & 32.5 & 32.5 \\
& 0.10 & 1.22 & 34.9 & 40.5 \\
& 0.01 & 1.47 & 39.1 & 47.8 \\
\hline
\end{tabular}

${ }^{a}$ Risk level. A risk level of 0.37 corresponds to the long-run minimal net cost. A risk level of $0.37,0.1$, or 0.01 corresponds to a probability of $0.37,0.1$, or 0.01 , respectively, that $C_{M K}$ will be exceeded in a given year.

${ }^{\mathrm{b}}$ Long-run expected Mycosphaerella graminicola related costs at the given dose.

c Costs which will be exceeded in a given year with probability $K$. yield loss (Appendix). As a numerical example of a change in the yield loss model, we examined loss relationships $g(S)$ that related the yield loss to the square root of disease severity:

$$
Y_{L}(D, S)=L \times S^{0.5} \times f(D)
$$

Equation 16 represents situations in which either physiology or market factors make small disease severity disproportionately damaging, as may be the case for $M$. graminicola in some settings (13) or for pathogens of horticultural crops, where low levels of blemish may render produce unmarketable.

\section{RESULTS}

None of the fits of the distribution frequency of severity in different seasons differed significantly from an exponential distribution. The parameter of the best-fitting exponential distribution differed between the resistant and susceptible cultivar groupings (Fig. 1; Table 2). Using equations 4 and 5, the probability distribution of disease severity was transformed into a probability distribution of net cost at a range of fungicide doses (Fig. 2). The standard deviation in costs decreases greatly with increasing fungicide dose, because the probability of extremely high costs decreases. For each fungicide dose shown in Figure 2, the cost cannot be less than the cost of that dose. Costs higher than this arise because of disease-induced yield losses. The probability of incurring a particular cost decreases as that cost increases because, for M. graminicola, more-severe epidemics are less probable than less-severe epidemics. A low dose is less effective at controlling disease, and the probability of substantial yield loss is greater than at higher dose; therefore, the probability of substantial costs is greater at lower than at higher doses.

Using the cumulative form of the exponential probability distribution, a function was derived for the net cost of disease which will be exceeded only in a specified proportion $K$ of years (equation 11). Equation 12 was used to find the dose which minimizes this cost given the grower's choice of $K$. To clarify the meaning of $K$, we can think of the grower deciding to accept disease-related costs above a specified level in "high-loss" years of high severity,

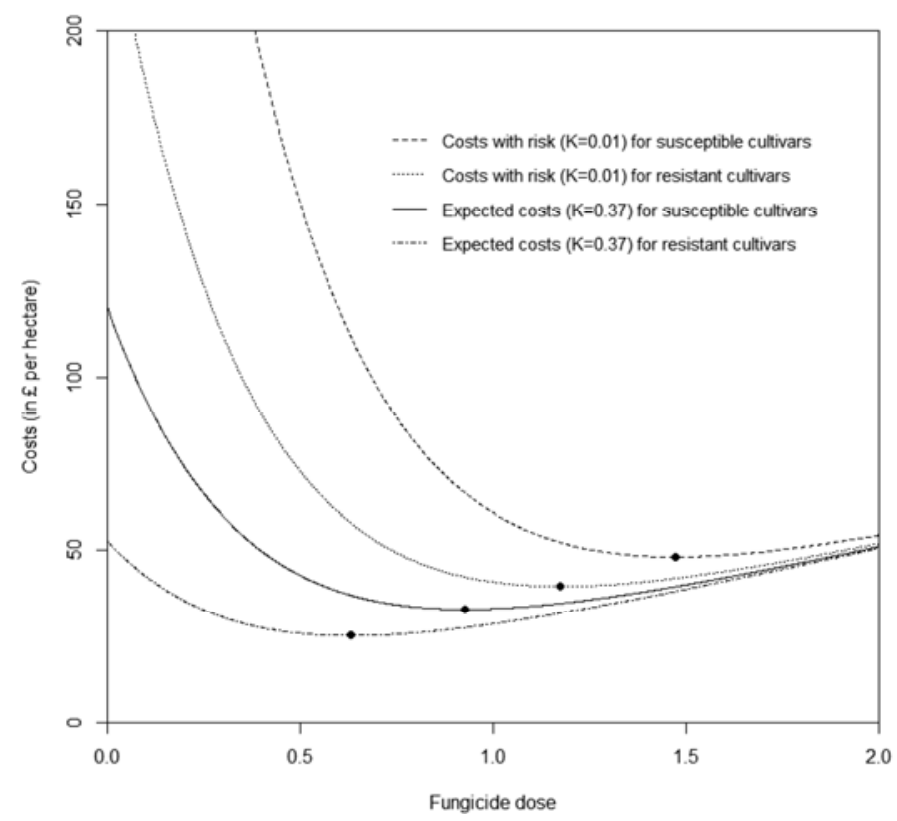

Fig. 4. Expected costs and costs which will not be exceeded with probability $K=0.10$ versus the total fungicide dose applied during the season for both resistant and susceptible cultivars (Table 2). The marks the optimum dose. Cost analyses were done using units of British pounds. These values were converted to American dollars at an exchange rate of 1 pound $=1.60$ dollars. 
which will occur in a proportion $K$ of years. The dose applied each year will then be calculated to minimize costs in the remaining years $(1-K)$.

Increased risk aversion increases the optimal fungicide dose and, for plausible values of $K$, the effect is considerable (Fig. 3). For example, if the acceptable risk to a grower for resistant cultivars is increased from $K=0.37$ to $K=0.10$, then the optimal fungicide dose increases from 0.6 to 0.9 (Table 5). A further decrease in acceptable risk to $K=0.01$ leads to a fungicide dose of 1.2 .

The absolute differences in optimal dose at different values of $K$ differ substantially between the resistant and susceptible cultivar groups, due to differences in disease severity distribution (Fig. 1; Table 2). Differences between cultivars and risk levels become smaller if the fungicide dose is increased further (Figs. 3 and 4). For all cost curves (Figs. 3 and 4), costs increase faster below the optimum dose than above it. The increase in costs below the optimum dose is the result of yield loss. The increase in costs above the optimum dose is the result of the cost of fungicide.

In the sensitivity analysis (Table 3 ), we show how our results are influenced by selected changes in the parameter values. Increases or decreases in the price of wheat $\left(P_{W}\right)$, production $(P)$, yield loss $(L)$, or price of a fungicide dose $\left(P_{D}\right)$ cause changes in the optimal fungicide dose. However, the form of the cost curves and the dose needed to achieve a certain risk reduction are unchanged (equation 15). When dose response parameter $k$ is reduced (so that the change in severity in response to a small reduction in dose from full is larger), the fungicide dose needed to achieve a certain risk reduction is increased. When the yield loss function is proportional to the square root of disease severity, thus giving disproportionately large losses at low severities, the optimal fungicide dose increases. The increase in fungicide dose needed to reduce the risk further decreases.

The general conclusion that a risk-averse grower will rationally adopt a higher dose than the dose which maximizes long-run profit holds under rather general conditions (Appendix).

\section{DISCUSSION}

Despite efforts to forecast epidemics, predictions of future disease severity made at the time of fungicide treatment decisions remain prone to considerable uncertainty $(14,15)$. As a consequence, growers have to make fungicide treatment decisions which account for the wide range of possible disease severities which may occur during the yield-forming period. The method introduced here shows how variation in disease severity can be quantified, translated into financial risk, and used to calculate an optimal fungicide dose that takes into account grower risk aversion. Increasing the fungicide dose can lead to a considerable reduction in risk and reduces the variability in the expected disease-related costs, in exchange for an insurance cost of lower long-term profit.

The analysis presented here is quite generally applicable to the control of diseases, weeds, and invertebrate pests by pesticide treatment. The numerical scenario studied was a relatively simple case involving one disease and one fungicide product. In commercial crops, growers often use formulated or tank mixtures of different active substances against a combination of diseases. The general principle and method of our analysis can be expanded to include more diseases and more fungicide products on a range of crops, provided data on severity distributions and fungicide dose responses are available. The concept of "dose" used here is quite general; in U.K. practice on wheat, it is literally the concentration of fungicide used at a predetermined spray point but, in other crops, it could refer to the spray interval.

The disease data used for the example in this article represent U.K. disease severities for M. graminicola over the years 2003 to 2005. For different regions or different periods in time, the disease probability distributions might differ. Although seasonal and local variation in disease severity $(S)$ is likely to be the predominant source of uncertainty, there are other parameters in the model that have a natural variation that was not studied here. There is natural variation in yield loss per unit disease $(L)$ $(7,13,16)$, fungicide dose response $\left(k\right.$ and $\left.R_{D}\right)$, and yield $(P)$. In Peterson et al. (12), the variances of such input parameters were combined using a Monte Carlo simulation. In a similar way, uncertainty in dose response, yield loss, and disease severity could also be combined, providing good estimates are available.

By associating a fungicide dose level with a risk level (chosen as a proportion of high-loss years, when higher-than-specified net costs will be accepted), decisions on what fungicide dose to use become more insightful. Instead of looking only at the financial cost and benefit of fungicide, a third aspect, risk and risk aversion, can also be taken quantitatively into account to optimize profit. The analysis here explains why growers use greater doses than might seem justified. An increased awareness of the relationship between dose and risk may ultimately lead to better decisions on the number of fungicide treatments and dose per treatment, and to more sophisticated advisory tools. If disease forecasting schemes or breeding for improved host resistance can constrain the probability distribution of disease severity, then the amount of fungicide applied as "insurance" could be substantially reduced.

\section{APPENDIX}

For simplicity, the main body of the text uses specific functions for $p(S), g(S)$, and $f(D)$. However, the results hold more generally, as we show here. The loss in a year with severity $S$ and fungicide $D$ is

$$
C_{M}(D, S)=D P_{D}+\operatorname{Lg}[S f(D)] \times P_{W} \times Y
$$

The critical level $S_{K}$ as a function of dose can be found by specifying a value for $\mathrm{MC}$ and rearranging equation $\mathrm{A} 1$, in the same way as equation 8 is derived from equation 5 . Inserting this value of $S_{K}$ into equation $\mathrm{A} 1$, differentiating with respect to $D$ and equating to zero gives

$$
-\left[P_{D} /\left(L \times P_{W} \times Y\right)\right]=[d / d D]\left\{\mathrm{g}\left[S_{K} f(D)\right]\right\}
$$

By the chain rule, and denoting differentiation by an apostrophe

$$
-\left(P_{D} /\left[L \times P_{W} \times Y \times f^{\prime}(D)\right]\right)=g^{\prime}\left[f(D) \times S_{K}\right]
$$

If $g$ is the identity function, so that loss is simply proportional to severity, as in the main text, then

$$
-\left(P_{D} /\left[L \times P_{W} \times Y \times f^{\prime}(D)\right]\right)=S_{K}
$$

By comparison, still assuming that $g$ is linear, the dose giving the highest average return is

$$
-\left(P_{D} /\left[L \times P_{W} \times Y \times f^{\prime}(D)\right]\right)=E(S)
$$

If $f^{\prime}$, the slope of the graph of disease severity against dose, decreases steadily with dose, then equation A4 will have at most one solution for $D$. If $S_{K}>E(S)$, the dose $D_{S}$ will be greater than the dose required to give the greatest average returns because $1 / f^{\prime}$ increases with $D$. That is, the dose required to give protection against a level of disease which should be exceeded only in a proportion $K$ of years (say 10\%) will be greater than that required to maximize average returns if that critical level is greater than the average level of disease. For plausible disease distributions and choices of $K$, this condition $S_{K}>E(S)$ will always be true. 
Fungicides which did not give decreasing disease with increasing dose over the practical range would never reach the market; thus, $f^{\prime}$ can be assumed to be negative. However, if $f^{\prime}$ initially increases at very low doses (because of removing competing organisms more than the pathogen, for example), then equations A4 and A5 would acquire more than one solution; however, the lower dose would represent a maximum rather than minimum cost. In the region around cost minima, the argument above would continue to hold.

If $g$ is not proportional to disease, it need not be true that the risk-averse dose will be greater than the dose giving the greatest average return. In particular, if loss sets in only at very high severity (for example, because of compensation, or a feature of the scoring system for the disease), then there could be a low probability (say 1\%) of very large losses. In that case doses which minimized average losses may be greater than the doses which ensured that a lower-but still critical-level of loss is exceeded only 1 in $1 / K$ years. However, if loss rises disproportionately with severity (as with diseases which cause cosmetic damage, such as Venturia inaequalis on apple) the contrary argument holds and the risk-averse dose may be much larger than the apparently economically optimal dose.

\section{ACKNOWLEDGMENTS}

Rothamsted Research receives support from Biotechnology and Biological Sciences Research Council of the United Kingdom. This project was partly funded by The Department for Environment Food and Rural Affairs, United Kingdom.

\section{LITERATURE CITED}

1. Anonymous. 2003-2005. HGCA recommended list of cereal varieties. Home-Grown Cereals Authority, London.

2. Finney, J. 1993. Risks and rewards from lower chemical inputs into agriculture. In: Proc. HGCA R\&D Conf. Home-Grown Cereals Authority, London.

3. Garthwaite, D. G., Thomas, M. R., Anderson, H., and Stoddart, H. 2004. Arable Crops in Great Britain: Pesticide Usage Survey Report 202. Central Science Laboratory, York, UK.
4. Gladders, P., Paveley, N. D., Barrie, I. A., Hardwick, N. V., Hims, M. J., Langton, S., and Taylor, M. C. 2001. Agronomic and meteorological factors affecting the severity of leaf botch caused by Mycosphaerella graminicola in commercial crops in England. Ann. Appl. Biol. 138:301311.

5. Hardwick, N. V., Jones, D. R., and Slough, J. E. 2001. Factors affecting diseases of winter wheat in England and Wales, 1989-98. Plant Pathol. 50:453-462.

6. King, J. E., Cook, R. J., and Melville, S. C. 1983. A review of Septoria diseases of wheat and barley. Ann. Appl. Biol. 103:345-373.

7. Paveley, N. D., Lockley, D., Sylvester-Bradley, R., and Thomas, J. 1997. Determinants of fungicide spray decisions for wheat. Pestic. Sci. 49:379388.

8. Paveley, N. D., Lockley, D., Vaughan, T. B., Thomas, M. R., and Schmidt, K. 2000. Predicting effective fungicide doses through observation of leaf emergence. Plant Pathol. 49:748-766.

9. Paveley, N. D., Sylvester-Bradley, R., Scott, R. K., Craigon, J., and Day, W. 2001. Steps in predicting the relationship of yield on fungicide dose. Phytopathology 91:708-716.

10. Paveley, N. D., Thomas, J. M., Vaughan, T. B., Havis, N. D., and Jones, D. R. 2003. Predicting effective doses for the joint action of two fungicide applications. Plant Pathol. 52:638-647.

11. Payne, R. W., Baird, D. B., Cherry, M., Gilmour, A. R., Harding, S. A., Kane, A. F., Lane, P. W., Murray, D. A., Soutar, D. M., Thompson, R., Todd, A. D., Wilson, G. T., Webster, R., and Welham, S. J. 2004. Genstat Release 8 Reference Manual, 3 volumes. VSN International Ltd., Oxford.

12. Peterson, R. K. D., and Hunt, T. E. 2003. The probabilistic economic injury level: incorporating uncertainty into pest management decisionmaking. J. Econ. Entomol. 96:536-542.

13. Shaw, M. W., and Royle, D. J. 1989. Estimation and validation of a function describing the rate at which Mycosphaerella graminicola causes yield loss in winter wheat. Ann. Appl. Biol. 115:425-442.

14. te Beest, D. E., Shaw, M. W., Paveley, N. D., and van den Bosch, F. 2009. Evaluation of a predictive model for Mycosphaerella graminicola for economic and environmental benefits. Plant Pathol. 58:1001-1009. Online publication. doi:10.1111/j.1365-3059.2009.02142.x

15. te Beest, D. E., Shaw, M. W., Pietravalle, S., and van den Bosch, F. 2009. A predictive model for early-warning of Mycosphaerella graminicola on winter wheat. Eur. J. Plant Pathol. 124:413-425.

16. Thomas, M. R., Cook, R. J., and King, J. E. 1989. Factors affecting development of Septoria tritici in winter wheat and its effect on yield. Plant Pathol. 38:246-257.

17. Wiik, L., and Rosenqvist, H. 2009. The economics of fungicide use in winter wheat in southern Sweden. Crop Prot. 29:11-19. 